\title{
Glass-Coated Microwires For Composites
}

\author{
Serghei A Baranov ${ }^{1,2 *}$ \\ ${ }^{1}$ Institute of Applied Physics, str. Academiei 5, Chisinau, MD-2028 Republic of Moldova \\ ${ }^{2}$ Shevchenko Pridnestrov'e State University, str. 25 Oktyabrya 128, Tiraspol, Republic of Moldova
}

*Corresponding author: Serghei A Baranov, Institute of Applied Physics, str. Academiei 5, Chisinau, MD-2028 Republic of Moldova, Shevchenko Pridnestrov'e State University, str. 25 Oktyabrya 128, Tiraspol, Republic of Moldova

\section{ARTICLE INFO}

Received: May 08, 2021

Published: 㸷 May 28, 2021

Citation: Serghei A Baranov. Glass-Coated Microwires For Composites. Biomed J Sci \& Tech Res 36(1)-2021. BJSTR. MS.ID.005804.

Keywords: Glass Coated Amorphous Micro and Nanowires; Taylor Ulitovsky Method

\begin{abstract}
In this paper, solutions for two problems are proposed. One of the problems is associated with increasing the strength of objects, for instance, the strength of windows in industrial buildings and dwelling houses. The other problem is related to electromagnetic shielding. Both of these problems are related to the protection form terrorist acts, since terrorists make use of concentrated electromagnetic pulses to destroy computers or other electronic equipment. The proposed solutions are based upon the manufacturing of glass windows reinforced with cast glass-coated amorphous micro- and nanowires (CGCAMNWs) having a special composition and structure, which increases their tensile strength against mechanical destruction, on the one hand, and imparts them with shielding properties against electromagnetic radiation, on the other hand. The CGCAMNW materials are of interest from both theoretical and practical points of view.
\end{abstract}

\section{Introduction}

One of the dangerous consequences of acts of terrorism is a traumatism of people owing to shattering of window glass of administration, industrial, and apartment houses. Therefore, an urgent problem is to improve the glass strength by decreasing the probability of forming and spreading the splinters during shattering. In addition, modern acts of terrorism can occur with use of concentrated beams of radio-frequency pulses in order to disable computers and other electronic devices, running of lifesupport systems of cities, etc. Furthermore, the radio-electronic intelligence service using directional electromagnetic radiation for reading information typed on the computer keyboard or displayed in the monitor, for example, via window opening, is concerned with modern acts of terrorism and espionage activity. It is known that conventional glass almost completely passes electromagnetic radiation in the entire frequency range. Therefore, the problem of producing radio-screening glasses is also urgent. The above problems can be solved in complex with the use of glass reinforced with glass-coated microwires prepared by the solution casting technique of the Taylor Ulitovsky method [1-4]. The reinforcement of glass with glass-coated microwires using an adhesive film increases the durability of the glass under shock and static loadings and prevents splinter scattering in the case of glass shattering.

In addition, this glass considerably reduces the transitivity of electromagnetic radiation in a wide frequency range-from a few hundreds of megahertz to a few tens of gigahertz. The reinforcement of glass with microwires does not reduce the light transmission ability in the entire gamut of colors, as in conventional glass, and does not worsen the transparency of the glass. Glasscoated microwires are almost imperceptible. Another possible application of microwires in the antiterrorist purposes, also for providing a hardening and screening effect, is the reinforcement of vests and helmets made of plastic, such as Kevlar, with microwire elements. The microwire represents a construction composed of a continuous metal core coated with a continuous glass coating. For a more precise comparison of the theory with the experiment, a set of experimental measurements is required; they are also discussed in the paper. The resulting microwires with an optimum chemical composition were tested for reinforcing window glass. A grid of high strength microwires was preconstructed using linear and orthogonal winding. After that, a melt was poured into special 
molds to reinforce window glass to obtain a sheet blank with a thickness of about $1-3 \mathrm{~mm}$.

\section{Casting of Glass-Coated Amorphous Magnetic Microwires}

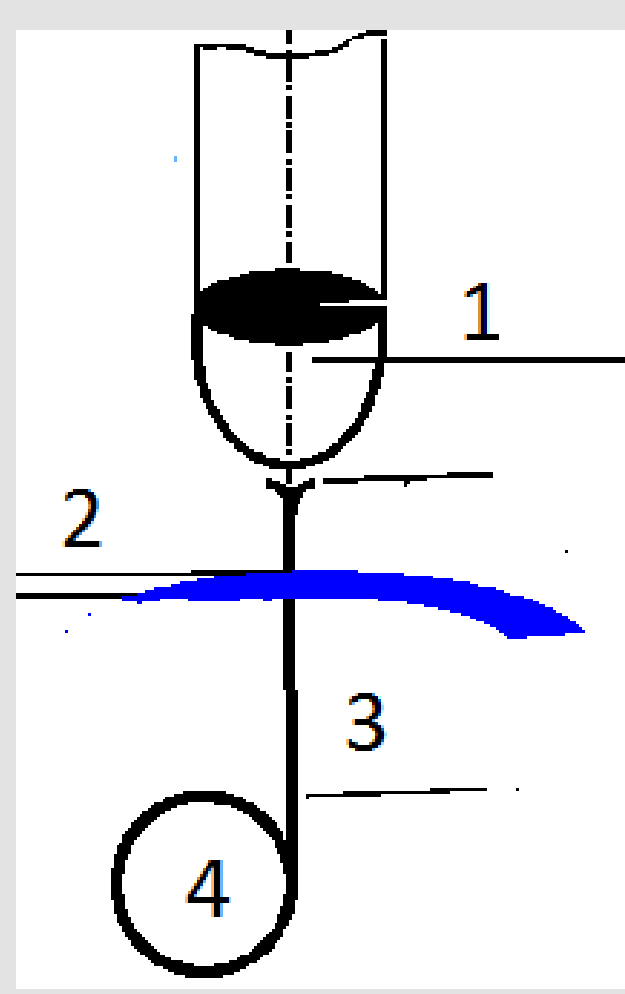

Figure 1: Casting of CGCAMNWs (see [1-4] and below):

(1) Glass tube with a metal droplet,

(2) Water,

(3) Glass-coated microwire, and

(4) Rotating receiving bobbin

Cast glass-coated amorphous micro- and nanowires (CGCAMNWs) are prepared using a rapid solidification technique, the so-called quenching and drawing procedure, or a modified Taylor Ulitovsky method [1-4], as shown in (Figure 1). In this process, an alloy in an amount of a few grams is placed inside a glass tube held directly over suitable heating means, for example, a highfrequency inductor heater. The alloy is heated up to the melting point to form a droplet. The portion of the glass tube adjacent to the melting metal softens to envelope the metal droplet. A glass filament is drawn from the softened glass portion and collected onto a receiving bobbin. Under certain drawing conditions, the molten metal can fill the glass capillary; thus, a microwire in which a metal core is covered continuously with glass is formed. The glass consumption in the process is compensated by continuous delivery of the glass tube in the inductor zone, whereas the formation of the metallic core is restricted to the initial amount of the droplet. The microstructure and, accordingly, properties of a microwire considerably depend on the cooling rate, which can be controlled by a cooling mechanism when the metal-filled capillary passes through a stream of a cooling liquid (water or oil) on its way to the receiving coil. The main advantages of this method for the production of cast glass-coated microwires are as follows [1-4]:

a) The formation of continuous long pieces of a microwire up to $104 \mathrm{~m}$ (in the case of a drip process). For a continuous process (see Figure 1), the microwire length is not limited.

b) A wide range of variations in the geometric parameters (typically the metallic core diameter $\mathrm{Dm}$ is in a range of $0.5-$ $70 \mu \mathrm{m}$, and the glass-coating thickness is in a range of $1-50 \mu \mathrm{m}$ ).

c) The control and adjustment of the geometric parameters (inner metallic core diameter Dm and glass thickness) during production.

d) The reproducibility of the physical properties and geometric parameters of the microwires in large-scale production.

\section{Production of Glass Reinforced with Microwires}

Glass reinforced with a microwire represents a three-layered construction consisting of two pieces of glass glued using a special adhesive film. The adhesive film consists of an adhesive base on two sides of which pieces of a microwire are put in mutual perpendicular directions. In the case of an electromagnetic wave incident on the interface between two media, a portion of the field is reflected from the surface; another portion permeates and spreads inside the other medium; the third portion interacts with the medium and is absorbed (transformed into heat). The coefficient of screening of the medium $\left|G_{\text {eff }}\right|$ can be written as follows:

$$
\left|G_{e f f}\right|=p_{r} / p_{s},
$$

where $\mathrm{P}_{\mathrm{r}}$ is the power of the incident wave and $\mathrm{P}_{\mathrm{s}}$ is the power of the past wave. Since an electromagnetic wave contains electrical and magnetic components, the interaction of an electromagnetic wave and a medium can be electrical in the case of a conducting medium and magnetic in the case of a screen with a high magnetic permeability.

a) For screening household and working buildings, inside which electromagnetic radiation is not located, for constructions and devices requiring protection against external electromagnetic radiation, it is reasonable to apply reflecting screens containing a microwire made of conducted materials (copper, silver, and alloys based on them). Depending on the screened object, the screens can be pliable and elastic, such as fabrics containing a microwire, and rigid, such as plastics, polymers, and glass or paper products.

b) For screening household and working buildings, in which, in addition to protection from external radiation, it is required to inhibit (weaken) electromagnetic radiation and reradiation from an internal source of radiation, it is reasonable to apply microwire-reinforced reflecting-absorbing screens having a 
high impedance at the working frequencies of the source. For example, in buildings with a powerful source operating at an extremely high radiation frequency, it is reasonable to apply microwire-reinforced materials having a resonant absorption frequency of the working source.

c) It is reasonable to use reflecting-absorbing multilayered electromagnetic shields for screening people (service personnel) working under conditions of high-level electromagnetic radiation.

d) Description of the design and technology of production of shielding. To provide functionality in electromagnetic screens, a microwire should be located as a grid construction. In this case, the microwire is located in a plane of the screen in two mutually perpendicular directions. The grid construction steps and the types and number of microwires in a construction are determined from requirements for the level of loosening the radiation power, the frequency range of screening, and expediency of applying the shielding. In implementing the stage of working out the technology for the preparation of triplex glass reinforced with microwires, technological equipment and process modes for gluing the glasses will be developed [5-7].

\section{Absorption Properties}

The design of GCAMNW composites was described in [8-11]. We have following typical configurations: Natural ferromagnetic resonance (NFMR) occurs if the sample is subjected to a microwave field without application of any biasing field other than the anisotropy field of the microwire [8-13] (Figures 2 \& 3).

Permeability dispersion is as follows:

$$
\mu(\omega)=\mu^{\prime}(\omega)+i \mu^{\prime \prime}(\omega) .
$$
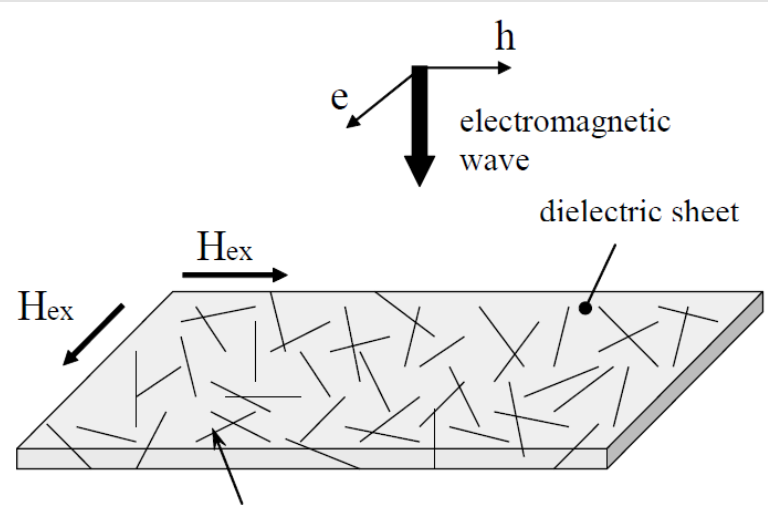

short wire inclusions embedded into the dielectric matrix

Figure 2: Composite shielding for radio absorption protection with GCAMNWs made with a stochastic mixture of microwires in the polymeric matrix.

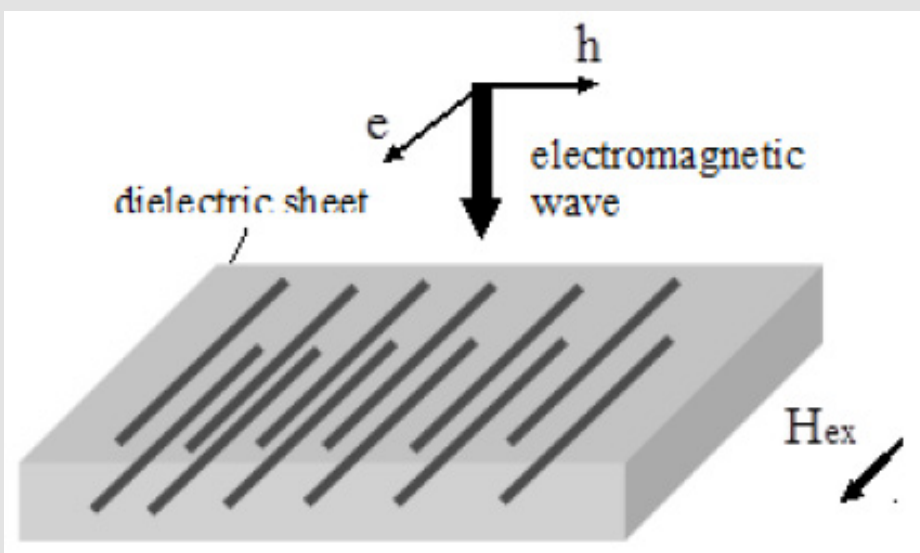

Figure 3: Composite shielding for radio absorption protection with GCAMNWs in the form of a grating.

Figures 4-6 show resonance frequencies of 7.5, 8.5, 10.5, and $13.5 \mathrm{GHz}$ and resonance widths of $1.5,2,3$, and $4 \mathrm{GHz}$. Near $\mu$ " resonance is expected to be described as follows:

$$
\mu^{\prime \prime} / \mu_{d c} \sim \Gamma \Omega /+\left[(\Omega-\omega)^{2}+\Gamma^{2}\right]
$$




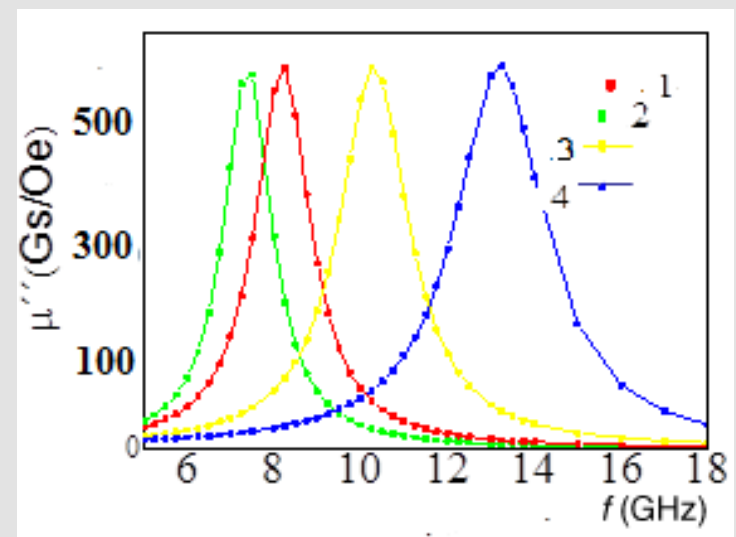

Figure 4: Imaginary relative permeability of components around NFMR for

(1) $\mathrm{CO}_{70} \mathrm{Fe}_{5}, 5 \mathrm{~B}_{14}, 5 \mathrm{Si}_{10}$

(2) $\mathrm{CO}_{70} \mathrm{Mn}_{7} \mathrm{~B}_{13} \mathrm{Si}_{10}$

(3) $\mathrm{CO}_{59} \mathrm{Fe}_{15} \mathrm{~B}_{16} \mathrm{Si}_{10}$, and

(4) $\mathrm{Fe}_{69} \mathrm{C}_{5} \mathrm{~B}_{16} \mathrm{Si}_{10}$ microwires.
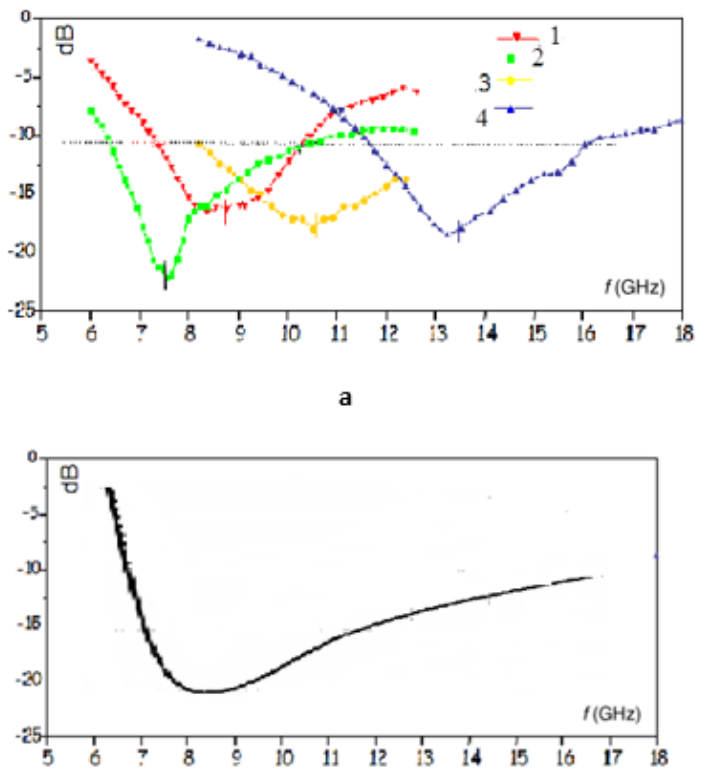

b

Figure 5:

a. Absorption characteristics of shieldings by a microwire composite of components around NFMR for

(1) $\mathrm{CO}_{70} \mathrm{Fe}_{5}, 5 \mathrm{~B}_{14}, 5 \mathrm{Si}_{10}$

(2) $\mathrm{CO}_{70} \mathrm{Mn}_{7} \mathrm{~B}_{13} \mathrm{Si}_{10}$

(3) $\mathrm{CO}_{59} \mathrm{Fe}_{15} \mathrm{~B}_{16} \mathrm{Si}_{10}$ and

(4) $\mathrm{Fe}_{69} \mathrm{C}_{5} \mathrm{~B}_{16} \mathrm{Si}_{10}$ microwires (see [5-11]).

b. Summary absorption characteristics of shielding by composite in an HF field of components around NFMR for

(1) $\mathrm{CO}_{70} \mathrm{Fe}_{5}, 5 \mathrm{~B}_{14}, 5 \mathrm{Si}_{10}$

(2) $\mathrm{CO}_{70} \mathrm{Mn}_{7} \mathrm{~B}_{13} \mathrm{Si}_{10}$

(3) $\mathrm{CO}_{59} \mathrm{Fe}_{15} \mathrm{~B}_{16} \mathrm{Si}_{10}$, and

(4) $\mathrm{Fe}_{69} \mathrm{C}_{5} \mathrm{~B}_{16} \mathrm{Si}_{10}$ microwires (see [5-11]). 


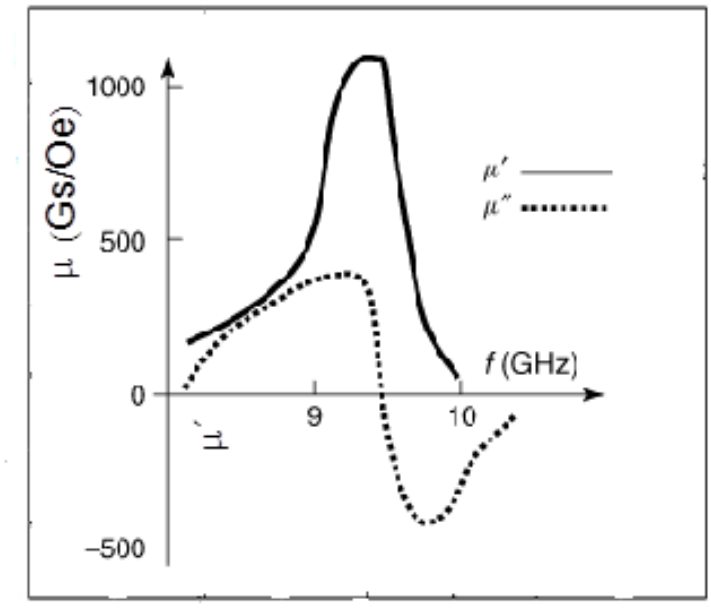

Figure 6: Frequency dispersion of the real and imaginary parts of relative permeability around the NFMR frequency for the $\mathrm{Fe}_{68} \mathrm{C}_{4} \mathrm{~B}_{16} \mathrm{Si}_{10} \mathrm{Mn}_{2}$ microwire (see [1,2,5-13]).

where $\mu_{\mathrm{dc}}$ is the static magnetic permeability and $\Gamma$ is the width of the resonant curve. Very near resonance, where $\Gamma>(\Omega-\omega)$, Eq. (3) reduces to

$$
\mu^{\prime \prime} / \mu_{d c} \sim \Omega / \Gamma \sim\left(10 \div 10^{2}\right)
$$

Monitoring the geometry of the microwire (i.e., wire diameter) and the magnetostriction through the microwire composition makes it possible to prepare microwires with desirable permeability dispersion and for absorption materials:

a) Determining the resonant frequency in a range of $1-12 \mathrm{GHz}$ and

b) Controlling the maximum of the imaginary part of magnetic permeability.

Figure 7 also shows the frequency absorption spectrum of shielding with $\mathrm{Fe}_{69} \mathrm{C}_{5} \mathrm{~B}_{16} \mathrm{Si}_{10}$ microwires. The varying attenuation is attributed to the lack of an ideal angular distribution of microwires, the length of which does not always fit into the shielding thickness. Pieces of microwires were embedded in planar polymeric matrices to form a composite shielding for radio absorption protection. Experiments were performed employing a commercial polymeric rubber with a thickness of about 2-3mm. Microwires were spatially randomly distributed over the matrix before its solidification. The concentration was maintained below 8-10 g of microwire dipoles (1-3mm long) per $100 \mathrm{~g}$ of rubber [1,5-7]. A typical result obtained in an anechoic chamber is shown in Figure 6 for shielding with embedded $\mathrm{Fe}_{69} \mathrm{C}_{5} \mathrm{~B}_{16} \mathrm{Si}_{10}$ microwires. It is evident that an absorption level of at least $10 \mathrm{~dB}$ is obtained in a frequency range of $8-12 \mathrm{GHz}$ with a maximum attenuation peak of $30 \mathrm{~dB}$ at about $10 \mathrm{GHz}$. In general, optimum absorption is obtained using microwires. It is evident that an absorption level of at least $10 \mathrm{~dB}$ is obtained in a frequency range of $8-12 \mathrm{GHz}$ with a maximum attenuation peak of $30 \mathrm{~dB}$ at about $10 \mathrm{GHz}$. In general, optimum absorption is obtained using microwires with metallic nuclei with a diameter of $2 r=1-3 \mu m(2 R \sim 20 \mu m(x>10))$. and a length of $\mathrm{L}=$ $1-3 \mathrm{~mm}$. These pieces of microwires can be treated as dipoles whose length $\mathrm{L}$ is comparable to the half value of effective wavelengths $\Lambda_{\text {eff }} / 2$ of the absorbed field in the composite material (i.e., in connection to a geometric resonance) (Figure 8).

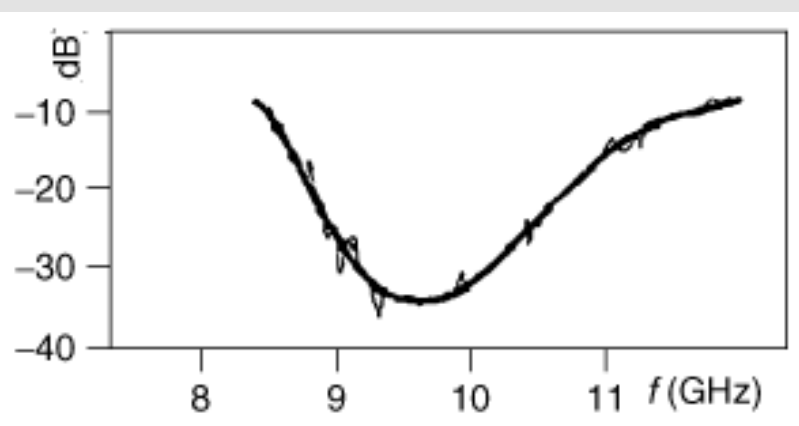

Figure 7: Typical absorption characteristics of shielding by a microwire composite with NFMR in an HF field in a frequency range of $10 \mathrm{GHz}$.

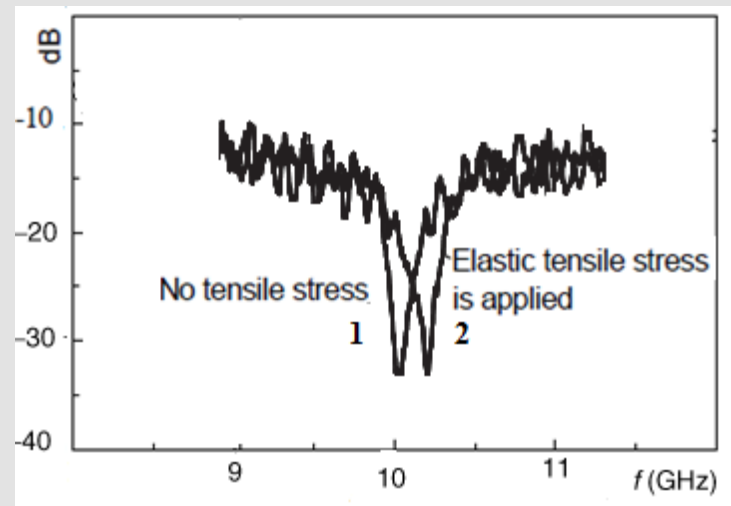

Figure 8:

(1) Average absorption characteristics of a shielding containing a microwire composite exhibiting NFMR in a microwave frequency range of $10-10.2 \mathrm{GHz}$ for $\mathrm{Fe}_{68} \mathrm{C}_{4} \mathrm{~B}_{16} \mathrm{Si}_{10} \mathrm{Mn}_{2}$ microwires ([2, 5-12]) and

(2) Absorption curve in the case of an external pressure (see [5-12]).

\section{Theory for Absorption Materials}

The propagation of an electromagnetic wave through absorption shielding with microwire-based elements is characterized by transmittance $|\mathrm{T}|$ and reflectance $\left|\mathrm{R}_{\mathrm{r}}\right|$ (coefficients given in [2, 5-13]):

$$
|T|=\left(\alpha^{2}+\beta^{2}\right) /\left[\left(1+\alpha^{2}\right)+\beta^{2}\right] ;\left|R_{r}\right|=1 /\left[\left(1+\alpha^{2}\right)+\beta^{2}\right],
$$


where $\alpha=2 \mathrm{X}_{\mathrm{r}} / \mathrm{Z}_{0}$ and $\beta=2 \mathrm{Y} / \mathrm{Z}_{0}$, with $\mathrm{Z}_{0}=120 \pi / \mathrm{Q}$, and the complex impedance $\mathrm{Z}=\mathrm{X}_{\mathrm{r}}+\mathrm{iY}$.

Absorption function $\mathrm{G}$ is correlated with the generalized highfrequency complex conductivity $\Sigma$ (or high-frequency impedance Z). Here, we use the analogy between the case of a conductor in a waveguide and that of a diffraction grating. The absorption function given by

$$
|G|=1-|T|^{2}-\left|R_{r}\right|^{2}=2 \alpha /\left[\left(1+\alpha^{2}\right)+\beta^{2}\right]
$$

has a maximum

$$
\left|G_{m}\right|=0.5 \geq|G|,
$$

for simultaneous $\alpha=1$ and $\beta=0$, for which

$$
|T|^{2}=\left|R_{r}\right|^{2}=0.25
$$

The minimum $|G|=0$ occurs at $\alpha=0$ and $\beta$ of any positive number

Theoretical estimations taking into account only the active resistance of microwires result in attenuation in a range of $5-10 \mathrm{~dB}$, which is much lower than experimental results, which for a spacing of microwires of $\mathrm{Q}=10^{-2} \mathrm{~m}$ ranges between 18 and $15 \mathrm{~dB}$, while for a spacing of $\mathrm{Q}=10^{-3} \mathrm{~m}$ it increases up to $20-40 \mathrm{~dB}$. Thus, it becomes clear that shielding exhibits anomalously high absorption factors, which cannot be attributed solely to the resistive properties of microwires.

Let us consider the effective absorption function [2, 5-13]:

$$
\left|G_{e f f}\right| \sim \Gamma_{e f f} \Omega_{e f f} /\left[\left(\Omega_{e f f}-\Omega\right)^{2}+\Gamma_{e f f}^{2}\right]
$$

where $\Gamma_{\text {eff }} \geq \Gamma$ and $\Omega \sim \Omega_{\text {eff }}=2 \pi \mathrm{c} / \Lambda$.

A microwave antenna will resonate when its length $\mathrm{L}$ satisfies to the condition

$$
L \sim \wedge / 2\left(\mu_{e f f}\right)^{1 / 2} .
$$

Absorptions maximum (see Figure 7) occurs for $\Omega_{\text {eff }} \sim 10 \mathrm{GHz}(\Lambda$ $\sim 3 \mathrm{~cm})$ and $\mu_{\text {eff }} \sim 10^{2}[2,5-13]$

This corresponds to

$$
L \sim 1.5-2 \mathrm{~mm},
$$

where the microwire concentration is much less than the percolation threshold. A higher concentration of dipoles leads to an increase in absorption $\left|G_{\text {eff }}\right|$ and an increase in reflectance $\left|R_{r 1}\right|$, which can be simply estimated as [2, 5-13]:

$$
\left|R_{r 1}\right| \sim 1-2 \sqrt{\left(\Omega / 2 \mu \Sigma_{m}\right)}
$$

where $\Omega / 2 \pi \sim 10^{10} \mathrm{~Hz}$
The formula is applicable, and calculation of small reflectance $\left|\mathrm{R}_{\mathrm{r} 1}\right|$ is possible, only if

$$
\Sigma_{m} \sim 10^{11} H Z
$$

for concentration below the percolation threshold (as $\left.\Sigma_{2} \sim 10^{15} \mathrm{HZ}\right)$

\section{Conclusion}

Microwave electromagnetic response has been analyzed for composites consisting of dipoles and a diffraction grating of amorphous magnetic glass-coated microwires in a dielectric. These materials can be employed for radio absorbing screening. The spontaneous NFMR phenomena observed in glass-coated microwires has opened the possibility of developing novel broadband radio absorbing materials.

The described studies provide the following basic conclusions [8]:

a) Cast GCAMNWs exhibit NFMR whose frequency depends on the composition, geometrical parameters, and deformation of the microwire. The NFMR phenomenon observed in glasscoated magnetic microwires opens up the possibility of developing new radio-absorbing materials with a wide range of properties. An important feature of cast microwires with an amorphous magnetic core is the dependence of the NFMR frequency on the deformation (stress effect). The calculations have shown that the shift of the NFMR frequency caused by the stress effect achieves $20 \%$ before the degradation of the composite.

b) The general technology of magnetic wire composites is costeffective and suitable for large-scale applications.

Here, the electromagnetic properties of composites with magnetic wires showing NFMR phenomena have been discussed. A striking property of these materials is that the spectra of the effective electromagnetic parameters (permittivity and permeability) can be actively tuned. The technology of glass coated amorphous microwires provides the preparation of continuous wires.

\section{Acknowledgment}

This work was supported by the Moldavian National project and the Shevchenko Pridnestrov'e State University project.

\section{References}

1. Baranov SA, Larin VS, Torcunov VT (2017) Technology, Preparation and Properties of the Cast Glass-Coated Magnetic Microwires. Crystals 7(6): 136.

2. Baranov SA (2017) An Engineering Review about Microwire, Lambert (Academic publishing)

3. HX Peng, Qin F, Phan MH (2016) Ferromagnetic Microwire Composites: From Sensors to Microwave Applications, Springer, Switzerland. 
4. Qin F, HX Peng (2013) Ferromagnetic microwires enabled multifunctional. composite materials. Progr Mater Sci 58(2): 183-259.

5. Baranov SA, M Yamaguchi, KL Garcia, M Vazquez (2008) Ferromagnetic Resonance in Cast Microwires and its Application for The Non-Contact Diagnostics. Surf Engin Appl Electrochem 44(6): 245.

6. Baranov SA (1998) Use of a microconductor with natural ferromagnetic resonance for radio-absorbing materials. Tech Phys Lett 24: 549-550.

7. Baranov SA (2015) Mold J Phys Sci 14 (3-4): 201.

8. E Adar, AM Yosher, S Baranov (2020) Natural ferromagnetic resonance in cast microwires and its application to the safety control of infrastructures. J Phys Res Appl 3: 118-122.

\section{ISSN: 2574-1241}

DOI: $10.26717 / B J S T R .2021 .36 .005804$

Serghei A Baranov. Biomed J Sci \& Tech Res

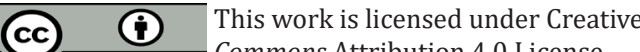

Submission Link: https://biomedres.us/submit-manuscript.php
9. E Adar, SA Baranov, NA Sobolev, AM Yosher (2020) Mold J Phys Sci 19(12): 89.

10. SA Baranov (2021) Ferromagnetic Glass-Coated Micro and Nanowires for Medical Application. Biomed J Sci Tech Res 32(5): 25413.

11. SA Baranov (2021) The Global Journal of Science Frontier Research. Global J Sci Front Res A 20(14).

12. MJ Malliavin, Acher 0, Boscher C, Bertin F, VS Larin (1999) chapter 5 giant magnetoimpedance. J Magn Magn Mat 420: 196-197.

13. AN Antonenko, SA Baranov, Larin VS (1997) electromagnetic wave absorbing material based on magnetic microwires. J Mat Sc and Eng, pp. 248.

$\begin{array}{ll}\text { BIOMEDICAL } & \text { Assets of Publishing with us } \\ \text { RESEARCHES } & \text { - Global archiving of articles } \\ \text { - Immediate, unrestricted online access }\end{array}$

